\title{
ION CHANNELS
}

\section{A paddle in oil}

\author{
Anthony G. Lee
}

\section{How do voltage-gated ion channels in cell membranes open? The latest work suggests that the process depends on having the correct lipid molecules in the membrane, with phosphate groups being mandatory.}

Electrical signalling in the nervous system involves voltage-gated ion channels, proteins that sit in the outer membrane of nerve cells. This membrane, like all membranes, consists of lipid molecules that are organized in the form of a bilayer - the charged parts of the molecules, the lipid headgroups, lie on the outside of the bilayer in contact with water, with the fatty acyl chains of the lipid molecules occupying the centre and forming the oily core. Embedded in this lipid bilayer are the voltage-gated ion channels. On page 775 of this issue, Schmidt, Jiang and MacKinnon ${ }^{1}$ show that having the correct lipids in the membrane is essential if the ion channels are to open when the voltage changes across the membrane*.

The voltage change that opens an ion channel - about $50 \mathrm{mV}$ - seems quite small, but a voltage change of $50 \mathrm{mV}$ across a membrane $50 \AA$ thick corresponds to a change of about $100,000 \mathrm{~V} \mathrm{~cm}^{-1}$, easily enough to cause a change in the structure of an ion channel. Our views of what exactly this structural change might be altered dramatically when MacKinnon's group determined the $\mathrm{X}$-ray structure of a voltage-gated ion channel ${ }^{2,3}$. The part of the ion channel that senses the change in voltage across the membrane is highly positively charged and is loosely attached to the outer face of the channel, exposed to the lipid bilayer. Because of its shape, MacKinnon referred to this part of the channel as a paddle (Fig. 1). The idea is that a change in voltage across the membrane results in movement of the paddle, which in turn leads to opening of the central pore of the channel, allowing ions to flow through the pore. It is currently a matter of debate whether the paddle moves all the way across the lipid bilayer when the voltage changes or whether its motion is more restricted ${ }^{4}$.

It seems likely that a bilayer of the correct lipid composition is essential for proper function of the paddle, because the paddle is exposed to the bilayer and has to move in it. The idea that the lipids are important has now been investigated by MacKinnon and colleagues ${ }^{1}$ in a very direct way. They used the traditional approach of forming lipid-bilayer membranes across a small hole in a plastic partition, and then inserting a voltage-gated

'This article and the paper concerned' were published online on 29 Nowember 2006 ion channel into the membrane by fusing vesicles containing the purified channel with the membrane. This allowed them to measure the current flowing through the ion channel as a function of the lipid composition of the membrane. For their experiments, they used the voltage-gated potassium channel from the bacterium Aeropyrum pernix because this can be expressed in high quantities in Escherichia coli and then purified.

The lipid composition chosen by MacKinnon and colleagues ${ }^{1}$ for their initial experiments was $70 \%$ phosphatidylethanolamine and $30 \%$ phosphatidylglycerol. This is the lipid composition of $E$. coli cell membranes, and the ion channel behaved perfectly in a bilayer of these lipids.

But what happens when the lipid composition is changed? The authors looked at the importance of phosphatidylethanolamine. Although this is the most abundant lipid in the $E$. coli membrane, it is rather unusual in that it does not, on its own, form bilayers at normal temperatures. Rather, because the overall shape of the molecule is conical, it tends to pack in three dimensions to give curved, nonbilayer structures. However, the presence in the membrane of a cylindrically shaped lipid molecule such as phosphatidylglycerol forces the phosphatidylethanolamine to adopt a bilayer structure, with the latter molecules being said to exist in the bilayer in a state of 'curvature frustration. There has been much speculation as to the possible significance of this frustrated state ${ }^{5}$. However, the authors found that channel function was unaltered when phosphatidylethanolamine was substituted with the cylindrically shaped, bilayer-favouring lipid phosphatidylcholine. So it seems that curvature frustration is not important for ionchannel function.

MacKinnon and colleagues then turned to the effect of phosphatidylglycerol, a lipid molecule with a negatively charged (anionic) headgroup. Anionic lipids would be expected to bind strongly to the positively charged paddle; it has been shown ${ }^{6}$, for example, that negatively charged lipids bind with high affinity to a positively charged patch on the mechanosensitive channel MscL. But the voltage-gated potassium channel was functional in the absence of phosphatidylglycerol, soany interaction between anionic lipids and the paddle cannot be essential for channel function.

Rather, it turns out that what is essential for function is the presence of a negatively charged phosphate group in the lipid molecule. The channel was totally inactive in non-natural, positively or negatively charged lipid molecules lacking a phosphate group, or in an uncharged, sugar-based lipid. It is intriguing that the membrane lipids of $A$. pernix, from which the channel originally came, are, unusually, all anionic, phosphate-containing lipids ${ }^{7}$.

Why is the phosphate group vital? MacKinnon and colleagues suggest that negatively charged phosphate groups in the lipid molecules may act as counterions for the positively charged arginine amino-acid residues in the paddle, stabilizing the charged paddle in the lipid bilayer. If this idea is correct, it would provide another example of the importance for membrane-protein function of the local interactions between a membrane protein and its surrounding lipid molecules.

Anthony G. Lee is in the School of Biological

Sciences, University of Southampton,

Southampton SO16 7PX, UK.

e-mait: agl@soton.ac.uk

1. Schmidt, D, Jiang Q-X.\&. MacKinnon, R. Nature 444, $775-779$ (2006).

2. Jiang, Y.et al. Nature 423, 33-41 (200B).

3 Long, S. B, Campbell, E. B. \&.MacKinnon, R. Science 309, 897-903 (2005).

4. Tombola, F, Pathak, M. M. \& lsacoft, E Y. Neuron 48, 719-725 (2005).

5. Lee, A. G. Blochim. Biophys Acta 1666,62-87 (2004)

6. Pow, A. M, East, 1.M.\& Lee, A.G. Biochemistry 44, 5873-5883 (2005).

7. Morili, Het al. Blachim. Biaphys. Acta 1436, 426-436 (1999) 